\title{
LAS DISCREPANCIAS OFICIALES: LA INDEPENDENCIA DE KOSOVO Y LA RETÓRICA EN EUROPA OCCIDENTAL ${ }^{1}$
}

\author{
Branislav Radeljić ${ }^{2}$ \\ School of Social Sciences, University of East London
}

\begin{abstract}
Resumen:
Este artículo examina los enfoques y las discrepancias oficiales que caracterizan la retórica de Europa occidental con respecto a la cuestión del estatuto de Kosovo. Desde los principios de la década de 1980, Kosovo ha sido cada vez más presente en los debates europeos, la tendencia que culminó con la intervención internacional de 1999 en la región y las negociaciones posteriores sobre su estatuto definitivo. Aunque los albaneses de Kosovo proclamaron su independencia en febrero 2008 y la mayoría de los Estados miembros de la UE decidieron reconocer Kosovo como un Estado independiente, la retórica de Europa occidental ha Estado bastante dividida. Este artículo muestra que, además de cinco miembros de la UE que han decidido no reconocer Kosovo desde el principio, y por lo tanto son suficientemente potentes para afectar a su progreso, tanto a nivel local como a nivel internacional, algunos de los Estados que lo han reconocido, a pesar de haber abandonado la política de "normas antes del estatuto", también se han esforzado por desarrollar su pleno apoyo a la provincia - una discrepancia que seguramente cuestiona el apoyo de Occidente en general por la independencia de Kosovo.
\end{abstract}

Palabras clave: La independencia de Kosovo, serbios, los albaneses de Kosovo, Unión Europea.

Title in English: "Official Discrepancies: Kosovo's Independence and Western Europe's Rhetoric"

Abstract:

This article examines approaches and official discrepancies characterising Western European rhetoric with regard to the Kosovo status question. Since the early 1980s, Kosovo has been increasingly present in European debates, culminating with the 1999 international intervention in the region and subsequent talks about its final status. Although the Kosovo Albanians proclaimed independence in February 2008 and the majority of EU Member States decided to recognise Kosovo as an independent state, Western European rhetoric has been rather divided. This article shows that in addition to five EU members who have decided not to recognise Kosovo from the very beginning, and thus are powerful enough to affect its further progress, both locally and internationally, some of the recognisers, although having abandoned the policy of "standards before status", have also struggled to develop full support for the province - a discrepancy that surely questions the overall Western support for Kosovo's independence.

Keywords: Kosovo independence, Serbs, Kosovo Albanians, European Union.

Copyright (C) UNISCI, 2014.

Las opiniones expresadas en estos artículos son propias de sus autores, y no reflejan necesariamente la opinión de UNISCI. The views expressed in these articles are those of the authors, and do not necessarily reflect the views of UNISCI.

\footnotetext{
${ }^{1}$ La versión original de este artículo fue publicada en inglés en la revista Perspectives on European Politics and Society, vol. 15, no. 4 (2014), pp. 431-444. El autor agradece a Andrija Daković por la traducción del texto al español.

${ }^{2}$ Branislav Radeljić es Profesor Asociado de Política Internacional en la Universidad de East London. Él es el autor de Europe and the Collapse of Yugoslavia: The Role of Non-state Actors and European Diplomacy (London, I. B. Tauris, 2012), y editor de Europe and the Post-Yugoslav Space (Farnham, Ashgate, 2013) y Debating European Identity: Bright Ideas, Dim Prospects (Oxford, Peter Lang, 2014).

Dirección: School of Social Sciences, University of East London, 4-6 University Way, Docklands, E16 2RD London, United Kingdom.

Email: B.Radeljic@uel.ac.uk.
} 


\section{Introducción}

Desde la actuación de la OTAN en la entonces República Federal de Yugoslavia en 1999, los países occidentales en general se han inclinado a ofrecer la provincia sureña serbia de Kosovo un status de país independiente que a menudo se presenta como el único camino a seguir. Tanto los funcionarios de Bruselas y Washington han insistido en que los albaneses de Kosovo merecen tener su propio Estado, debido a las atrocidades cometidas por los dirigentes serbios en las últimas décadas. Tras la declaración unilateral de 2008 de independencia de Kosovo, la persistencia de dos expectativas opuestas dentro de la comunidad internacional que Kosovo será reconocido tan pronto como sea posible o que nunca se convertirá en un Estado independiente - ha llevado a la creciente confusión y la decepción sobre el status final de Kosovo. El hecho de que la provincia sigue no estando "ni aquí ni allá" es capaz de afectar a su propia relevancia, así como al progreso regional, especialmente con respecto a la adhesión a la Unión Europea.

Aunque Occidente a menudo ha sido percibido por los líderes serbios como siendo proalbanés en el tema de Kosovo, sin embargo Occidente no está unido totalmente en las discusiones sobre el status de Kosovo, su situación económica, el clima político, la pertenencia a las instituciones internacionales, las estrategias y la legislación para abordar los problemas sociales, etc. Las discrepancias que caracterizan a la retórica oficial de Europa occidental no sólo existen entre los Estados que apoyan la independencia de Kosovo y los que ponen objeciones a la misma (como es el caso en la Unión Europea, con 23 de sus Estados miembros que han reconocido el Estado de Kosovo y cinco - Chipre, Grecia, Rumania, Eslovaquia y España - que han rechazado hacerlo), sino también entre los Estados que fueron los primeros en reconocerlo.

En este artículo se muestra la existencia de la tendencia anteriormente mencionada, examinado las declaraciones oficiales e informes (sobre la situación en Kosovo y la decisión de reconocerlo) y, más precisamente, esbozando las discrepancias existentes entre los principales representantes de los Estados y sus respectivos departamentos gubernamentales (por ejemplo, en el Reino Unido, Alemania e Italia). En consecuencia, después de un breve resumen de la entrada y la presencia de Kosovo en la retórica de Europa Occidental (a través de debates en el Parlamento Europeo), el artículo se centra en los debates posteriores a 1999 y la argumentación, enfatizando en particular las discrepancias oficiales posteriores a 2008. Aparte de considerar este enfoque como muy problemático, dada su capacidad de incidir en el progreso internacional y la reputación de Kosovo, el artículo propone la realización de dos preguntas importantes: “¿Hasta qué punto los países de Europa Occidental y Europa Occidental, en general, apoyan que Kosovo sea un Estado independiente?” y “Cuáles son las implicaciones de este inconsistente enfoque occidental?"

\section{Kosovo en la retórica de Europa Occidental}

Los archivos de las instituciones de la Unión Europea ofrecen una importante colección de documentos sobre las relaciones entre la entonces Comunidad Económica Europea y la Federación Yugoslava. Sobre la base de las transcripciones disponibles de debates en el Parlamento Europeo, Kosovo fue mencionado por primera vez en 1981, a raíz de disturbios anteriores, comenzando en la Universidad de Pristina, y la escalada en los enfrentamientos armados entre los estudiantes albaneses y la policía de la provincia ${ }^{3}$. Durante el debate, un miembro del Parlamento Europeo pidió "a los Ministros de Relaciones Exteriores de los

\footnotetext{
${ }^{3}$ Ver, por ejemplo: Artisien, Patrick F. R. \& Howells, R. A.: "Yugoslavia, Albania and the Kosovo Riots", The World Today, vol. 37, no. 11 (1981), pp. 419-427.
} 
Estados miembros reunidos en la cooperación política que expresaran su preocupación al gobierno yugoslavo y presionaran para que la sección de la población albanesa de Yugoslavia tuviera garantizada la igualdad de oportunidades para el desarrollo en los ámbitos económicos, sociales, culturales y estructurales"4. Algunos debates posteriores también expresaron preocupaciones similares. ${ }^{5}$ De hecho, a finales de la década de 1980, los europeos se habían dado cuenta de las diferencias y las tensiones existentes dentro de la federación yugoslava. En Yugoslavia, las repúblicas de Eslovenia y Croacia, preocupadas por el futuro del Estado reorganizado por Tito y frustrados por la obligación de mantener a sus regiones menos desarrolladas, ya habían promovido sus propios contactos con Occidente - un proyecto que se desarrolló aún más debido a la subida de Slobodan Milošević y el creciente dominio del régimen serbio ${ }^{6}$. En Kosovo, cuya autonomía fue revocada en 1989 y muchos profesionales albanokosovares destituidos de sus cargos, la situación se volvió aún más compleja, induciendo una política de resistencia pacífica y la creación de instituciones paralelas no oficiales. Tras el estallido de la crisis yugoslava y las declaraciones de independencia de Croacia y Eslovenia en junio de 1991, los albaneses de Kosovo decidieron celebrar un referéndum no oficial en septiembre, para garantizar su propia independencia, y elecciones no oficiales en mayo de 1992 dando lugar a la proclamación de la República de Kosovo ${ }^{7}$.

Teniendo en cuenta que la participación europea en el espacio yogoslavo en la década de 1990 se centró principalmente en los sucesos de Eslovenia, Croacia y en los más problemáticos de Bosnia y Herzegovina, la cuestión de Kosovo parecía más bien marginal. El hecho de que los representantes de la Unión Europea no utilizaran los Acuerdos de Paz de Dayton de 1995 para abordar también el status de autonomía de Kosovo fue un asunto grave de preocupación entre los albaneses de Kosovo, interpretándose como un desinterés europeo en hacer frente a sus necesidades ${ }^{8}$. Por lo tanto, dejar sin resolver la cuestión de Kosovo representó una oportunidad para que el Ejército de Liberación de Kosovo criticase e hiciera caso omiso de la doctrina pacifista de la Liga Democrática de Kosovo - un enfoque que generó una serie de enfrentamientos entre albaneses locales y fuerzas serbias. La falta de un enfoque impulsado por el grupo pro bono Public International Law and Policy, a finales de 1998 (que sugería comenzar con una soberanía intermedia, que se caracterizaba por una reducción gradual de control serbio sobre la provincia de Kosovo, que a su vez permitiría a la población local adquirir autoridad soberana, capaz de proteger los intereses legítimos de la minoría serbia, y terminar con un referéndum sobre la independencia y la búsqueda de reconocimiento internacional) y la escalada del conflicto en enero de 1999 (cuando las fuerzas serbias cometieron un crimen contra la humanidad por la muerte de más de cuarenta civiles en la aldea de Račak, en el centro de Kosovo) ${ }^{9}$ eran indicadores claros de que iba a requerirse

\footnotetext{
${ }^{4}$ Parlamento Europeo, "Motion for a resolution”, 2 de julio de 1981, Bruselas, Los archivos históricos de las instituciones de la UE.

5 Parlamento Europeo, "Debates of the European Parliament: Yugoslavia", 8 de marzo de 1983, Bruselas, Los archivos históricos de las instituciones de la UE; Parlamento Europeo, "Resolution on the situation in Kosovo", 13 de abril de 1989, Bruselas, Los archivos históricos de las instituciones de la UE.

${ }^{6}$ Radeljić, Branislav (2012): Europe and the Collapse of Yugoslavia: The Role of Non-State Actors and European Diplomacy, London and New York, I. B. Tauris.

${ }^{7}$ Krieger, Heike (ed.) (2001): The Kosovo Conflict and International Law: An Analytical Documentation, Cambridge, Cambridge University Press, p. 522.

${ }^{8}$ Laakso, Liisa: "A Capability-Implementation Gap in the Making? Multi-Level Governance and European Crisis Management”, en Walzenbach, Günter P. E. (ed.) (2006): European Governance: Policy Making between Politicization and Control, Aldershot, Ashgate, pp. 147-165; 152; Sell, Louis (2002): Slobodan Milošević and the Destruction of Yugoslavia, Durham, Duke University Press, p. 274; Toje, Asle (2008): America, the EU and Strategic Culture: Renegotiating the Transatlantic Bargain, Oxon, Routledge, p. 52.

${ }^{9}$ Ver, por ejemplo: Bideleux, Robert \& Jeffries, Ian (2007): The Balkans: A Post-Communist History, Oxon, Routledge, p. 542; Gordy, Eric (2013): Guilt, Responsibility and Denial: The Past at Stake in post-Milošević
} 
algún tipo de participación externa. En este punto, Occidente había llegado a ser plenamente consciente de su fracaso en la prevención en un primer momento, y luego en hacer frente a las consecuencias del genocidio de Srebrenica en 1995 y la división artificial de Bosnia y Herzegovina. Según Joschka Fischer, entonces ministro de Exteriores alemán, actuar cortésmente con los funcionarios de Belgrado sólo conduciría a más fosas comunes, por lo que afirmó que el uso de la fuerza debía ser considerado: "Yo no soy amigo de usar la fuerza, pero a veces es un medio de último recurso. Así que estoy dispuesto a usarlo si no hay otra vía de solución. Si las personas están siendo masacradas, no se puede murmurar diciendo que no exite un mandato. Hay que actuar" ${ }^{\prime 10}$. Este punto de vista fue aprobad e incluso llegó a hacerse oficial cuando la Secretaria de Estado de EE.UU., Madeleine Albright participó en las conversaciones de paz de Rambouillet, que tenían como finalidad la negociación de un acuerdo entre las dos partes enfrentadas, y subrayó: "Déjenme decirles que si las conversaciones fracasan porque los serbios no dicen 'Sí', tendremos que bombardear. Si las conversaciones fracasan debido a que los albaneses no dicen 'Sí', no vamos a ser capaces de apoyarlos $\mathrm{y}$, de hecho, tendremos que cortar toda la ayuda que están recibiendo desde el exterior" $" 11$.

La literatura existente sobre la guerra de Kosovo de 1999 se ha centrado en todo tipo de aspectos, incluyendo el contexto general ${ }^{12}$, la legalidad y las consecuencias de la participación de la OTAN en $1999^{13}$, las posiciones post-intervencionistas ${ }^{14}$, y la proclamación de la independencia y el reconocimiento internacional ${ }^{15}$. Sin embargo, los más recientes relatos han

Serbia, Philadelphia, University of Pennsylvania Press, p. 7; Kritsiotis, Dino: “The Kosovo Crisis and NATO's Application of Armed Force Against the Federal Republic of Yugoslavia”, International and Comparative Law Quarterly, vol. 49, no. 2 (2000), pp. 330-359.

${ }^{10}$ Fischer citado en Cohen, Roger: "Germany's Pragmatic Ex-Radical Thinks Globally", The New York Times, 28 de enero de 1999, en http://www.nytimes.com/1999/01/28/world/germany-s-pragmatic-ex-radical-thinksglobally.html.

${ }^{11}$ War in Europe: A Documentary (2000).

${ }^{12}$ Bieber, Florian \& Daskalovski, Židas (eds.) (2005): Understanding the War in Kosovo, London, Frank Cass; Buckley, Mary \& Cummings, Sally. N. (eds.) (2001): Kosovo: Perceptions of War and its Aftermath, New York, Continuum; Judah, Tim (2002): Kosovo: War and Revenge, New Haven, Yale University Press; Kostovicova, Denisa (2005): Kosovo: The Politics of Identity and Space, Oxon, Routledge; Mertus, Julie A. (1999): Kosovo: How Myths and Truths Started a War, Berkeley, University of California Press; Phillips, David L. (2012): Liberating Kosovo: Coercive Diplomacy and US Intervention, Cambridge, The MIT Press.

${ }^{13}$ Croft, Catherine \& Williams, Paul R.: "Was the Former 1999 NATO Intervention an Illegal War against the Former Republic of Yugoslavia?”, en Di Lellio, Anna (ed.) (2006): The Case for Kosova: Passage to Independence, London and New York, Anthem Press, pp. 121-128; Falk, Richard A.: "Kosovo, World Order and the Future of International Law", American Journal of International Law, vol. 93, no. 4 (1999), pp. 847-857; Glennon, Michael J.: "The New Interventionism: The Search for a Just International Law", Foreign Affairs, vol. 78, no. 3 (1999), pp. 2-7; Steinberg, James B.: "A Perfect Polemic: Blind to Reality on Kosovo", Foreign Affairs, vol. 78, no. 6 (1999), pp. 128-133; Wedgwood, Ruth: "NATO's Campaign in Yugoslavia", The American Journal of International Law, vol. 93, no. 4 (1999), pp. 828-834.

${ }^{14}$ Bacevich, Andrew J. \& Cohen, Eliot A. (eds.) (2001): War over Kosovo: Politics and Strategy in a Global Age, New York, Columbia University Press; Bellamy, Alex J. (2002): Kosovo and International Society, Basingstoke, Palgrave Macmillan; Daalder, Ivo H. \& O'Hanlon, Michael E. (2000): Winning Ugly: NATO's War to Save Kosovo, Washington, The Brookings Institution; Ker-Lindsay, James (2009): Kosovo: The Path to Contested Statehood in the Balkans, London and New York, I. B. Tauris; Lambeth, Benjamin S. (2001): NATO's Air War for Kosovo: A Strategic and Operational Assessment, Santa Monica, RAND; Latawski, Paul \& Smith, Martin A. (2003): The Kosovo Crisis and the Evolution of Post-Cold War European Security, Manchester, Manchester University Press; Tomuschat, Christian (2002): Kosovo and the International Community: A Legal Assessment, Alphen aan den Rijn, Kluwer Law International; van Ham, Peter \& Medvedev, Sergei (eds.) (2002): Mapping European Security after Kosovo, Manchester, Manchester University Press.

${ }^{15}$ Borgen, Christopher J.: "Advisory Opinion, Accordance with International Law of the Unilateral Declaration of Independence in respect of Kosovo", International Legal Materials, vol. 49, no. 5 (2010), pp. 1404-1440; Hilpold, Peter (ed.) (2012): Kosovo and International Law: The ICJ Advisory Opinion of 22 July 2010, Leiden, Brill; Perritt, Henry H. (2011): The Road to Independence for Kosovo: A Chronicle of the Ahtisaari Plan, 
hecho caso omiso de las consecuencias de las discrepancias que caracterizaron la retórica oficial de Europa Occidental. La actual falta de posición común entre los Estados miembros de la UE no sólo desafía las relaciones entre ellos, sino que también proporciona a las autoridades serbias una impresión o, más precisamente, la ilusión de que el caso de Kosovo podría tomar una dirección completamente diferente y terminar de forma favorable, como parte constitutiva de Serbia o, menos deseable, pero aún mejor que la independencia, como una provincia que se dividió entre los serbios y los albaneses de Kosovo. Además, varios departamentos gubernamentales de los países de Europa occidental que apoyan firmemente la independencia de Kosovo, han contribuido a una mayor complejidad general proporcionando a menudo información altamente desacreditativa sobre Kosovo que a su vez estimula toda una serie de preguntas con respecto al diseño de las políticas de la UE.

\section{De las "pautas de referencia antes de status" $" 16$ un status sin pautas de referencia}

Consciente de los posibles problemas que iba a traer consigo la puesta en práctica de la Resolución 1244, adoptada en junio de 1999, el Consejo de Seguridad de la ONU dio la bienvenida a "la labor iniciada por la Unión Europea y otras organizaciones internacionales para desarrollar un enfoque integral para el desarrollo económico y la estabilización de la región afectada por la crisis de Kosovo, incluida la aplicación de un Pacto de Estabilidad para el Sudeste de Europa, con amplia participación internacional a fin de fomentar la promoción de la democracia, la prosperidad económica, la estabilidad y la cooperación regional"17. Tal como aconteció, la UE convenció a la comunidad internacional para participar en el Pacto de Estabilidad, puso en marcha la Agencia Europea de Reconstrucción en 2000 e introdujo el Proceso de Estabilización y Asociación en el año 2001. La tarea principal de estas iniciativas fue hacer frente a la inestabilidad en los Balcanes y proporcionar un marco para la estabilización y el desarrollo futuro de la región.

En ese momento, Javier Solana, Alto Representante de la UE para la Política Exterior y de Seguridad Común, recordó a los albaneses de Kosovo que su independencia no estaba en el orden del día y que, técnicamente, Kosovo seguía siendo parte de Yugoslavia. Sostuvo que la función central de la presencia internacional era establecer las pautas de referencia primero y luego discutir el status final ${ }^{18}$. Sin embargo, la Misión de Administración Provisional de las Naciones Unidas en Kosovo (UNMIK), establecida por la Resolución 1244, no logró unificar los estándares políticos. El diálogo entre la UNMIK y las instituciones de Kosovo no fue productivo. Por ejemplo, el nombramiento de los jueces internacionales resultó insuficiente debido a las constantes presiones y amenazas que se derivaban de los extremistas en un ambiente predominantemente albanés, no estando dispuestos a cooperar en la búsqueda de los autores de delitos por motivos étnicos; de acuerdo con un informe posterior, sólo había 15 jueces internacionales y 10 fiscales internacionales que prestaban servicios en el sistema de

Cambridge, Cambridge University Press; Summers, James (ed.) (2011): Kosovo: A Precedent? The Declaration of Independence, the Advisory Opinion and Implications for Statehood, Self-Determination and Minority Rights, Leiden, Martinus Nijhoff Publishers; Weller, Marc (2009): Contested Statehood: Kosovo's Struggle for Independence, Oxford, Oxford University Press.

16 Traducción de "Standars before Status".

${ }^{17}$ Naciones Unidas, United Nations Security Council Resolution 1244, 1999, en http://daccess-dds-ny.un.org/doc/UNDOC/GEN/N99/172/89/PDF/N9917289.pdf?OpenElement.

${ }^{18}$ Radeljić, Branislav: "Europe and the Post-Yugoslav Space: From Intervention to Integration", en Radeljić, Branislav (ed.) (2013): Europe and the Post-Yugoslav Space, Farnham, Ashgate, pp. 1-32, p. 24. 
justicia local que solo era capaz de hacer frente a sólo el tres por ciento de los casos penales ${ }^{19}$. Además, como se había advertido a nivel internacional, "el cambio constante y la experiencia del personal en puestos clave socavaron la continuidad e interrumpieron la actuación en iniciativas claves" ${ }^{, 20}$.

Los dos aspectos que cuestionaban seriamente la simpatía general occidental por la independencia de Kosovo en este período incluyeron el enfoque de "las pautas de referencia antes del status", inaugurado por el tercer jefe de la UNMIK, Michael Steiner de Alemania ${ }^{21}$, aprobado por la UE y bien recibido por las autoridades serbias, y las expectativas que se perfilaron durante la Cumbre UE-Balcanes en Salónica, en junio de 2003, que se esperaba evaluase el potencial de la región y su futuro europeo. Aunque la política de " pautas de referencia antes del status" cubrió una amplia gama de temas, que iban desde el establecimiento de las instituciones democráticas y el Estado de derecho al desarrollo de la economía de mercado y el diálogo con las autoridades de Belgrado, su esencia "fue que exigía a las instituciones de autogobierno de Kosovo que demostrasen que estaban dispuestas y eran capaces de proteger los derechos de todas las comunidades étnicas de Kosovo, y tenían la capacidad para actuar de una manera civilizada" ${ }^{22}$. Poco después, la Cumbre UE-Balcanes en Salónica, al tiempo que señaló que el futuro de los Balcanes está en la Unión Europea, subrayó que esa condición sólo sería posible tras el cumplimiento de los mismos criterios utilizados para los Estados de Europa Central $^{23}$. El hecho de que en este momento los europeos habían comenzado a reemplazar el papel dominante de Estados Unidos en los Balcanes fue percibido de forma bastante general por los albaneses de Kosovo como la intención europea de suspender cualquier debate político sobre el status final de Kosovo y, lo que es más preocupante, como el apoyo a la posición de Serbia con respecto al status de Kosovo. Creyeron que la autonomía que habían conseguido como resultado de la Resolución 1244 era, de hecho, muy limitada ${ }^{24}$; y que, a pesar de que tuvieron lugar elecciones e Ibrahim Rugova, líder de la Liga Democrática de Kosovo, fue elegido presidente de Kosovo, lo que los albaneses realmente habían conseguido era una ilusión de autonomía ${ }^{25}$.

Una vez que la UE hubo incrementado su presencia, se había creado una expectativa general de que la UE jugaría un papel central en los intentos que determinan el estatuto futuro de Kosovo. Como sus representantes acordaron, todo el proceso se basa en los siguientes principios clave: "Kosovo no debe volver a la situación anterior a marzo de 1999 y Belgrado y Pristina deben avanzar hacia la integración euroatlántica; El status de Kosovo debe basarse en la diversidad étnica, la protección de las minorías, la protección del patrimonio cultural y religioso, y mecanismos eficaces de lucha contra la delincuencia organizada y el terrorismo; la solución del status de Kosovo debe reforzar la seguridad y la estabilidad regional; por

\footnotetext{
${ }^{19}$ Hartmann, Michael E.: "International Judges and Prosecutors in Kosovo: A New Model for Post-Conflict Peacekeeping", USIP Special Report, 13 de octubre de 2003, en http://www.usip.org/publications/internationaljudges-and-prosecutors-in-kosovo-new-model-post-conflict-peacekeeping.

${ }^{20}$ International Crisis Group, “Two to Tango: An Agenda for the New Kosovo SRSG”, Europe Report, no. 148 (3 de septiembre de 2003), en http://www.crisisgroup.org/en/regions/europe/balkans/kosovo/148-two-to-tangoan-agenda-for-the-new-kosovo-srsg.aspx.

${ }^{21}$ Steiner, Michael: "Speech to Donors' Conference", Bruselas, 5 de noviembre de 2002, en http://www.unmikonline.org/press/2002/pressr/pr864.htm.

${ }^{22}$ Ante, Arta (2010): State Building and Development: Two Sides of the Same Coin? Exploring the Case of Kosovo, Hamburg, Disserta Verlag, p. 150.

${ }^{23}$ Consejo: "EU-Western Balkans Summit", Thessaloniki, 21 de junio de 2003, en http://www.eu2003.gr/en/articles/2003/6/23/3135/index.asp.

${ }^{24}$ Mehmeti, Leandrit I.: "Democratization in Kosovo: The Role of International Institutions", en Radeljić, Branislav (ed.) (2013): Europe and the Post-Yugoslav Space, Farnham: Ashgate, pp. 183-209, pp. 197-198.

${ }^{25}$ Gallagher, Tom (2005): The Balkans in the New Millennium: In the Shadow of War and Peace, London and New York, Routledge, p. 154.
} 
consiguiente, no debe haber ningún cambio en el actual territorio de Kosovo (es decir, no partición de Kosovo y no unión de Kosovo con cualquier país o parte de otro país tras la resolución del status de Kosovo); cualquier solución debía ser plenamente compatible con los valores y las normas europeas y contribuir a la realización de la Perspectiva Europea sobre Kosovo y la región; Kosovo seguirá necesitando una presencia internacional civil y militar" ${ }^{26}$. La decisión de la UE de mostrar su posición apoyándose en numerosas obligaciones sólo sirvió para irritar aún más a las partes en conflicto. Sus dirigentes consideraron que la confianza entre ellos y la UE estaba perdiendo fuerza y que, mientras los albaneses de Kosovo tenían que tratar de deshacerse de la política de "pautas de referencia antes del status", los serbios estaban resucitando sus sentimientos nacionalistas, lo que conducía al deterioro de la cooperación con el Tribunal Penal Internacional para la antigua Yugoslavia y la suspensión de las negociaciones de adhesión con la UE en mayo de 2006.

La promoción de "pautas de referencia antes del status", a pesar de ser un intento ambicioso, fue completamente erosionada y abandonada después de los disturbios de mediados de marzo de 2004, y reemplazada con un Plan de Aplicación de las Normas, con un enfoque particular en la economía, estado de derecho y el diálogo entre Belgrado y Pristina. La comunidad internacional, mientras se colocaba entre las dos partes enfrentadas, entendía que las pautas de referencia no se podrían cumplir en el corto plazo, y, además, que la falta de status y de seguridad en Kosovo estaba afectando a toda la región de los Balcanes. Como un autor explicó, "desde el final del conflicto de Kosovo en el verano de 1999, la comunidad internacional ha lidiado con un dilema. No hay perspectivas de una estabilidad verdaderamente sostenible en la región, mientras el status de Kosovo no haya sido resuelto. Al mismo tiempo, abordar esta cuestión en sí misma presenta riesgos considerables para la estabilidad. Si la comunidad internacional aborda la cuestión del status sin la preparación adecuada, el resultado podía ser un punto muerto en el mejor de los casos y un enfrentamiento en el peor. Si se esperaba demasiado tiempo los elementos inestables que seguían persistiendo en el territorio, en particular, la impaciencia de la población de Kosovo, la persistente crisis económica y las relaciones aún tensas entre las comunidades étnicas bien podrían conducir a una nueva crisis"27.

$\mathrm{Si}$ analizamos las posiciones existentes en el momento, podemos afirmar que la comunidad internacional o, más precisamente, la Unión Europea, que a menudo se presentaba como un actor que había acumulado una gran destreza y conocimientos en toda la década de 1990, fomentando la integración de los Estados de Europa Central en sus estructuras. Al basarse en esta experiencia, el gobierno de Bruselas siguió insistiendo en los procesos de democratización y europeización, lo que para la gente del lugar, dado que el status de la provincia estaba pendiente, no tenía importancia alguna. En pocas palabras, la brecha entre los deseos promovidos a nivel internacional y las necesidades reales no podría dar lugar a las reformas estructurales, la prosperidad económica y el empleo, a pesar de que se reconoce ampliamente como una poderosa fuerza motivadora para la futura adhesión a la UE; en cambio, la provincia continuó conservando su imagen de agujero negro en los Balcanes, extremadamente corrupto e implicado en el crimen organizado transnacional. Con estos aspectos en mente, no es de extrañar que la literatura inmediatamente posterior a la crisis de Kosovo ha tendido a presentar el caso de Kosovo como una verdadera prueba para la UE, de la que se esperaba que proporcionase a la parte albanesa nada que no fuera la plena

\footnotetext{
${ }^{26}$ Consejo: "Summary Note on the Joint Report by Javier Solana, EU High Representative for the CFSP, and Olli Rehn, EU Commissioner for Enlargement, on the Future EU Role and Contribution in Kosovo", 14 de junio de 2005, en http://www.consilium.europa.eu/ueDocs/cms_Data/docs/pressdata/en/reports/85228.pdf.

${ }^{27}$ Lehne, Stefan: "Has the 'Hour of Europe' Come to Last? The EU's Strategy for the Balkans", en Batt, Judy (ed.) (2004): The Western Balkans: Moving On, Paris, Institute for Security Studies, pp. 111-124, p. 116.
} 
independencia de Kosovo y resolver la consecuente frustración serbia: "Es difícil ver qué participación en los asuntos de Kosovo, si existe alguna, quedaba a las autoridades de Belgrado. Si es posible mantenerla dentro de Serbia, solo puede ser por medio de una nueva versión de autonomía que permite a la provincia autónoma obtener la mayor parte de la sustancia de la independencia sin conseguir el status formal de reconocimiento diplomático y ser miembro de la ONU. Implica también que las fuerzas armadas del Estado al que están unidos, no tendrán acceso al territorio de la minoría" 28.

La retórica oficial de Europa occidental presentó las infames conversaciones de Viena de finales del 2005 como si tuvieran como objetivo resolver el status final de Kosovo ${ }^{29}$. Como la descentralización era considerada capaz de proporcionar mecanismos de protección de las minorías, los mediadores internacionales decidieron que el proceso de negociación entre los representantes serbios y albaneses de Kosovo debía comenzar con el tema de la descentralización - un paso importante, dado el fracaso anterior de un proyecto piloto para la creación de nuevos municipios en ciertas áreas con mayoría serbia ${ }^{30}$. La reunión de enero de 2006 que siguió fue un completo fracaso; como se observa después, "ninguna de las partes había cedido una pulgada", lo que significa que "las agencias de ayuda se preparan para la opción menos mala - otro éxodo de los serbios"31. En febrero, los mediadores no fueron capaces de reconciliar las posiciones de las dos partes sobre la cuestión de las reformas de los gobiernos locales; mientras que el lado serbio insistió en que la descentralización del gobierno se debe iniciar lo más pronto posible, los delegados de Kosovo mantuvieron que esto era posible sólo después de determinar el status de Kosovo. Durante una ronda aún más difícil de marzo, las partes continuaron con sus puntos de vista dispares sobre descentralización del gobierno en Kosovo. Hablaron de los problemas de la financiación comunitaria, la cooperación entre las comunidades y las relaciones de las comunidades serbias con Serbia. Además, el equipo serbio sugirió que las comunidades serbias debían financiarse, además de por los ingresos por impuestos locales, con cargo al presupuesto nacional de Serbia, mientras que los albaneses sostuvieron que tal arreglo financiero, al igual que cualquier otro tipo de ayuda extranjera, podría entrar en Kosovo sólo a través de gobierno central.

Los intercambios subsiguientes de puntos de vista se centraron en las fronteras comunes, instalaciones religiosas, las relaciones de propiedad, los problemas económicos, así como la necesidad de una presencia internacional después de la determinación del status de Kosovo. Aún así, las dos partes no quisieron cambiar sus posiciones adoptadas inicialmente. Por ejemplo, el primer ministro serbio, Vojislav Kostunica, subrayó que "la existencia de Kosovo y Metohija como parte de Serbia y la existencia del pueblo serbio en Kosovo son los objetivos clave de la participación de Serbia en las conversaciones políticas sobre el futuro estatuto de esa región", y que cualquier decisión sobre Kosovo "debe hacerse dentro de Serbia, en el marco de la gran autonomía de Kosovo y Metohija dentro de Serbia, mientras que cualquier otra decisión - ya sea descentralización del poder o el status de autonomía - son sólo sus especificaciones" ${ }^{32}$. Sin embargo, Kostunica afirmó que el gobierno serbio estaba

\footnotetext{
${ }^{28}$ Dent, Martin: "Lessons of Kosovo", en Waller, Michael, Drezov, Kyril \& Gökay, Bülent (eds.) (2001): Kosovo: The Politics of Delusion, London, Frank Cass, pp. 120-130, p. 124.

${ }^{29}$ Ver: Weller, Marc: "The Vienna Negotiations on the Final Status of Kosovo", International Affairs, vol. 84, no. 4 (2008), pp. 659-681.

${ }^{30}$ UNDP, "Early Warning Report: Kosovo", no. 12 (2006), en http://www.kosovo.undp.org/ publications/ews12/ewr12 eng.pdf.

${ }^{31}$ Hundley, Tom: "Wary Serbs Watch Deadlocked Talks on Kosovo's Independence", Chicago Tribune, 10 de junio de 2006, en

http://articles.chicagotribune.com/2006-06-10/news/0606100075_1_albanians-kosovo-independence.

32 “Vienna Talks: Albanians Don't Hurry to Recognise the Rights of Serbs in Kosovo", Regnum, 29 de marzo de 2006, en http://www.regnum.ru/english/613129.html.
} 
dispuesto a llegar a un compromiso en cierta medida y por lo tanto proporcionar a la provincia de Kosovo, "una gran autonomía - algo entre la independencia y la autonomía europea estándar". Por el contrario, el presidente de Kosovo, Fatmir Sejdiu, expresó la esperanza de su delegación "de que el proceso iniciado en Viena se terminaría rápidamente y con éxito para que este año [2006] se convirtiese realmente en un año para determinar el status de Kosovo de conformidad con la voluntad del pueblo - que es la independencia" 33 .

El resultado de las costosas y largas conversaciones de Viena, lo que podría haberse previsto cuando se hizo evidente que las partes involucradas no estaban dispuestas a transigir, confirmaron la pertinencia de algunos argumentos ofrecidos anteriormente que indican que una plena independencia de Kosovo no podía ser negociada, sino solo impuesta ${ }^{34}$. Sin embargo, la retórica de Europa Occidental en el momento - la decisión de Solana y de Rehn de lanzar una nueva misión, en virtud de la Política Común de Seguridad y Defensa de la UE, para proporcionar apoyo a Kosovo y asistencia en la realización de las reformas necesarias presentó a los europeos como interesados en la estabilización de la provincia y en la mejor preparación para su status futuro, mientras esperaban que las autoridades serbias se centrasen en el progreso en las negociaciones de adhesión a la UE en lugar de preservar Kosovo dentro de las fronteras serbias. El problema fue que la administración de Bruselas, si bien decidió ignorar la política lanzada por Alemania de "pautas de referencia antes de status", siguiendo adelante con la cuestión del status de Kosovo, no realizó ninguna oferta sustancial o, hablando con más propiedad, no ofreció ninguna indemnización compensatoria a la parte serbia, sino que continuó con su política de condicionalidad.

\section{2008: Proclamación de la independencia y período posterior}

La declaración de independencia de Kosovo en 2008 no debe ser percibida como una gran sorpresa, sobre todo teniendo en cuenta que varios miembros de la elite política postMilošević de Serbia, entre ellos el primer ministro posterior Zoran Djindjić, y algunos de sus seguidores más cercanos, hablaban de la provincia de Kosovo como de facto independiente y que Serbia tenía que centrarse en sí misma, es decir, de seguir adelante con los procesos de democratización y europeización. Sin embargo, la declaración fue inmediatamente cuestionada por el liderazgo de Serbia, lo que resulta en una solicitud oficial al Tribunal Internacional de Justicia (TIJ) para que emitiera una opinión consultiva sobre si la declaración unilateral de independencia de Kosovo era conforme al derecho internacional. Tanto la declaración y la consiguiente opinión del tribunal de que la iniciativa Kosovo-Albanesa en realidad no violaba el derecho internacional alentó la proliferación de una literatura inquieta por los aspectos legales y, de tanto o más importancia, por las implicaciones para el sistema internacional y los futuros intentos secesionistas ${ }^{35}$. Como señala correctamente un estudio, "el fallo del TIJ que constituye un as en las manos de los dirigentes políticos de Kosovo en su búsqueda del pleno reconocimiento de su independencia. También es un alivio para los Estados que han reconocido hasta ahora la independencia de Kosovo. Pero no hay señales

\footnotetext{
${ }^{33}$ Ibid.

${ }^{34}$ Oberg, Jan \& Mitić, Aleksandar, "Kosovo: Many Options but Independence”, 2005, en http://www.transnational.org/pressinf/ 2005/pi228_Oberg_Mitic_Kosovo.html.

${ }^{35}$ Borgen, Christopher J.: "Advisory Opinion, Accordance with International Law of the Unilateral Declaration of Independence in Respect of Kosovo", International Legal Materials, vol. 49, no. 5 (2010), pp. 1404-1440; Fierstein, Daniel: “Kosovo's Declaration of Independence: An Incident Analysis of Legality, Policy and Future Implications", Boston University International Law Journal, Issue 26 (2008), pp. 417-442; Hilpold, Peter (ed.) (2012): Kosovo and International Law: The ICJ Advisory Opinion of 22 July 2010, Leiden, Brill; Vidmar, Jure: "International Legal Responses to Kosovo's Declaration of Independence", Vanderbilt Journal of Transnational Law, vol. 42, no. 3 (2009), pp. 779-851.
} 
aún de que vaya a convencer a los otros Estados del sistema internacional que hasta ahora se han negado a reconocer a Kosovo" 36 .

En toda la UE, una gran mayoría de sus Estados miembros se apresuraron a reconocer la nueva República autoproclamada de Kosovo. Para empezar, el Reino Unido ignoró totalmente su posición adoptada anteriormente "describiendo las pautas de referencia para Kosovo como la única forma de avanzar hacia el status final", aclarando que "no había nada automático en el proceso. Si Kosovo hiciese los avances necesarios en el cumplimiento de las normas, se pasaría a la siguiente etapa. Pero si no, tendría que someterse a un nuevo examen"37. Aunque las conversaciones relacionadas con las pautas de referencia habían desaparecido y el gobierno británico apoyaba abiertamente la creación de un nuevo Estado, algunos de sus departamentos han continuado ofreciendo planteamientos más negativos sobre Kosovo, lo que genera claramente un dilema con respecto a la orientación general del Reino Unido respecto a la cuestión de Kosovo. Por ejemplo, la sección de la Oficina de Relaciones Exteriores y de la Commonwealth sobre los viajes y estancias en el extranjero advierte a los potenciales viajeros a Kosovo que la situación es "tensa", caracterizada por "un riesgo subyacente de terrorismo" e "incidentes esporádicos de violencia armada y explosiones de vehículos en las principales ciudades que por lo general están vinculados con el crimen organizado"38. Sin embargo, en caso de que todavía decidiesen ir allí, deben tratar de evitar Leposavić, Zvečan, Zubin Potok y la parte norte de la ciudad de Mitrovica, por lo tanto, las zonas dominadas por serbios, "debido a la violencia ocasional que existe allî" 39 . La opinión de la Oficina de Relaciones Exteriores corresponde en gran medida a la ofrecida por la Red24, un grupo de gestión de riesgos de seguridad global con sede en Londres, lo que sugiere "un alto grado de precaución" cuando se viaja a Kosovo, criticando el nivel existente de delincuencia organizada: "La corrupción y el mercado negro son actividades frecuentes en Kosovo, con los sindicatos del crimen bien establecidos dedicados a oficios lucrativos de narcóticos y armas, así como la trata de personas. La imposibilidad de establecer el imperio de la ley durante la transición del país a la independencia ha permitido a estos sindicatos desarrollar vínculos y entrar en colusión con las empresas comerciales legítimas, e incluso las principales instituciones del Estado y los titulares de cargos públicos. También hay fuertes conexiones entre los líderes del crimen organizado y la gran diáspora albanesa en otras partes del mundo, especialmente en la vecina Albania"40.

Al igual que los británicos, el gobierno alemán, a pesar de una conciencia clara de la escala de las actividades del crimen organizado y su efecto perjudicial en la estabilización de Kosovo después de $1999^{41}$, abandonó la idea de las pautas de referencia y se apresuró a reconocer la provincia sureña de Serbia como un Estado independiente. Al igual que el Ministerio de Asuntos Exteriores y de la Commonwealth, el Consejo de Asuntos Exteriores de Alemania Federal ha proporcionado casi la misma descripción y consejo a los viajeros potenciales o incluso los inversores, advirtiéndoles de que Kosovo es un país con muchas armas en manos privadas que se utilizan con facilidad, aunque, como se ha subrayado, no en

\footnotetext{
36 Noutcheva, Gergana (2012): European Foreign Policy and the Challenges of Balkan Accession: Conditionality, Legitimacy and Compliance, Oxon, Routledge, p. 108.

${ }^{37}$ Naciones Unidas, "Implementing 'Standards before Status' Policy Core Political Project for UN Kosovo Mission”, Security Council told, Press Release, 6 de febrero de 2004, SC/7999.

38 Foreign and Commonwealth Office, "Kosovo", 2013, en http://www.fco.gov.uk/en/travel-and-livingabroad/travel-advice-by-country/europe/kosovo.

${ }^{39} \mathrm{Ibid}$.

40 "Kosovo: Country Intelligence", Red24, 2013, en

http://www.red24.com/members/intelligence/kosovorepublic.php.

${ }^{41}$ Institut für Europäische Politik, "Operationalisierung von Security Sector Reform (SSR) auf dem Westlichen Balkan - intelligente/kreative Ansätze für eine langfristig positive Gestaltung dieser Region”, 2007. Berlin, IEP.
} 
contra de los extranjeros ${ }^{42}$. A lo que se añade la comprensión oficial italiana de la situación en Kosovo que es muy similar a las dos anteriores, dejando la impresión de que casi se debe evitar ir a Kosovo ${ }^{43}$.

En contraste con los citados Estados miembros de la UE, que han reconocido a Kosovo, pero se han mantenido bastante reservados sobre su arreglo definitivo y el progreso futuro, hay cinco miembros que han rechazado seguir la tendencia post-febrero 2008 de reconocer la independencia de Kosovo. Por ejemplo, Rumanía ha decidido permanecer firme y no poner en peligro su retórica inicial, defendiendo que "el Consejo de Seguridad no debería considerar dar un estatuto futuro de la provincia hasta que se hiciesen efectivos los estándares estipulados en la comunidad internacional. Las normas no eran meros puntos de referencia técnicos; constituían la medida de la transformación de Kosovo en una zona que ofrece seguridad y oportunidades para todos sus habitantes y en un área que ya no era una amenaza para la estabilidad regional. Lo que estaba en juego no era el status de una provincia, sino el de todos y cada uno de los miembros de su población" 44 .

Así, desde la perspectiva actual, es posible argumentar que la introducción y posterior abandono de la política de "las pautas de referencia antes del status" han generado una serie de cuestiones desconcertantes, tanto en la UE como a nivel local. En primer lugar, una política de este tipo subraya la existencia de incoherencias en la perspectiva global de la UE, dejando espacio para especulaciones de cómo y por qué ha sido imposible hablar con una sola voz. A pesar de que la mayor parte de los distintos gobiernos de los Estados miembros de la UE han reconocido a Kosovo, los Informes de Progreso de la UE posteriores a 2008 han retratado a Kosovo como extremadamente problemático - un aspecto que cuestiona de forma evidente el enfoque de los Estados que le han reconocido a nivel individual. Como uno de los informes observó, "Kosovo tiene que seguir avanzando en el establecimiento y la consolidación del Estado de Derecho y tiene que mejorar el funcionamiento y la independencia de su poder judicial. Es necesario establecer un historial en la lucha contra la corrupción, el lavado de dinero y el crimen organizado, que demuestre resultados concretos. Kosovo también necesita fortalecer la capacidad, la independencia y la profesionalidad de su administración pública y mejorar el entorno empresarial, incluyendo la regulación, la supervisión y la gestión empresarial. Kosovo tiene que garantizar una total transparencia en los nombramientos públicos de alto nivel y asegurarse de que la contratación pública cumple con los criterios de independencia, objetividad y transparencia. Kosovo debe establecer una política macroeconómica y fiscal sostenible... Kosovo tiene que mejorar la protección de los serbios y otras minorías y mejorar el diálogo y la reconciliación entre las comunidades" ${ }^{\text {45 }}$. Del mismo modo, los informes posteriores exponen toda una serie de problemas, que van desde "debilidades en las políticas fiscales y de gasto y, en aplicación de la ley, incluida la lucha contra la corrupción y la delincuencia organizada"46 a la falta de capacidad "para mejorar la aplicación del marco jurídico vigente y la ejecución de resoluciones para corregir las violaciones de los derechos humanos" ${ }^{, 47}$.

\footnotetext{
42 Federal Foreign Office: “Kosovo: Reise und Sicherheitshinweise", 2013, en http://www.auswaertigesamt.de/DE/Laenderinformationen/00-SiHi/KosovoSicherheit.html.

${ }^{43}$ Farnesina: "Viaggiare sicuri: Kosovo", 2013, en http://www.viaggiaresicuri.it/?kosovo.

${ }^{44}$ Naciones Unidas: "Implementing 'Standards before Status' Policy Core Political Project for UN Kosovo Mission”, Security Council told, Press Release, 6 de febrero de 2004, SC/7999.

${ }^{45}$ Comisión Europea: "Communication from the Commission to the European Parliament and the Council: Kosovo* - Fulfilling its European Perspective", Bruselas, 14 de octubre de 2009, COM(2009)5343, p. 5.

${ }^{46}$ Comisión Europea: "Kosovo* 2011 Progress Report", Bruselas, 12 de octubre de 2011, SEC(2011)1207, p. 31.

${ }^{47}$ Comisión Europea: “Kosovo* 2013 Progress Report”, Bruselas, 16 de octubre de 2013, SWD(2013)416, p. 14.
} 
En segundo lugar, esta política confusa proporcionó a los Estados miembros de la UE que no habían reconocido a Kosovo, la oportunidad de prevenir o, al menos, posponer los intentos secesionistas en casa. Teniendo en cuenta diversos debates, su posición ha sido criticada ampliamente por los 23 Estados que sí le habían reconocido: por ejemplo, Ulrike Lunacek, un miembro del Parlamento Europeo de Austria fue muy lejos al afirmar que los cinco (que no habían reconocido) deben ser forzados a reconocer a Kosovo, pero luego claramente esbozó diversos problemas que se pueden utilizar como una justificación de por qué no hacerlo: "La relativa debilidad del gobierno de Kosovo, después de las elecciones fraudulentas y un proceso prolongado en la votación y toma de posesión del nuevo presidente y la formación de gobierno en la primavera de 2011, así como las denuncias sobre el crimen organizado formuladas por el informe de Dick Marty en la Asamblea Parlamentaria del Consejo de Europa, no proporcionan al primer ministro Thaçi una sólida posición en la discusión y el poder que iba a necesitar para mostrar la fuerza necesaria en el ámbito diplomático y doméstico",48.

A nivel local, todo el discurso "las pautas de referencia antes del status" ha irritado a los albaneses de Kosovo, ya que entendieron que su situación no se iba a resolver tan rápidamente como se había pensado inicialmente. Las declaraciones oficiales del gobierno de Bruselas, así como los distintos gobiernos de la UE han apoyado la presencia de la misión Estado de Derecho en Kosovo de la UE (EULEX), debido a los riesgos de la escalada del conflicto - una iniciativa bien acogida por los albaneses locales. Sin embargo, el hecho de que sus resultados fueron bastante limitados, sobre todo respecto a la policía, la justicia y los asuntos aduaneros ${ }^{49}$, hizo muy difícil que los albaneses se identificaran con un Estado propio e independiente. Como Ilir Deda, el director del Instituto Kosovar de Investigación y Desarrollo de Políticas, ha dicho, "hace cinco años, nadie habría podido creer, que en la actualidad Kosovo no sería un miembro de la ONU, que no íbamos a tener un equipo de fútbol nacional y que estaríamos atrapados en las negociaciones con Serbia... Mucha gente piensa que a Kosovo no se le ha dado una oportunidad justa todavía. Puede ser la pertenecía a la UEFA [Unión de Asociaciones Europeas de Fútbol], el Festival de la Canción de Eurovisión o el Consejo de Europa - la cuestión es que Kosovo sigue sentado en la sala de espera de casi todas las organizaciones internacionales" ${ }^{\text {. }}$.

Por fin, los dirigentes serbios, plenamente conscientes de las posiciones existentes a nivel internacional, así como la de los albaneses de Kosovo, han percibido que la política "pautas de referencia antes de status" es una política válida sobre la que insistir y con la que podrían tratar de posponer la solución global del status de Kosovo. Más importante aún, el hecho de que Kosovo no tenga un puesto en la ONU o que no haya sido reconocido por todos los Estados miembros de la UE se ha interpretado como una oportunidad adicional, resultando posiblemente en un plan B. Vale la pena recordar que al comienzo de las conversaciones sobre el status, serbios y albaneses de Kosovo se presentaron con un plan sólo A, lo que significaba que uno de ellos saldría como un ganador absoluto y el otro como un perdedor absoluto. La comunidad internacional ha desempeñado un papel importante en la formación de dicho enfoque que no incluye ningún compromiso. Por ejemplo, se excluyó la partición basándose en el hecho de que ello implicaría traslados forzados de población en las zonas norte y sur de la provincia, posiblemente acompañado de numerosos enfrentamientos y

\footnotetext{
${ }^{48}$ Lunacek, Ulrike: “Kosovo-Serbia: Will They ever Come to Terms? What is the European Union's Role?", en Džihić, Vedran \& Hamilton, Daniel (eds.) (2012): Unfinished Business: The Western Balkans and the International Community, Washington, Center for Transatlantic Relations, pp. 149-157, p. 151.

49 Greiçevci, Labinot: "EU Actorness in International Affairs: The Case of EULEX Mission in Kosovo", Perspectives on European Politics and Society, vol. 12, no. 3 (2011), pp. 283-303.

50 "Kosovo ist als Staat noch Stückwerk", Die Zeit, 14 de febrero de 2013, en http://www.zeit.de/politik/ausland/2013-02/kosovo-5-jahre-unabhaengigkeit.
} 
víctimas, y podría ser mal utilizada como un método de solución de conflictos, capaz de incentivar la redefinición de las fronteras en la región en otros lugares, sobre todo en Bosnia y Herzegovina. Al final, el enfoque de "pautas de referencia antes del status" era demasiado para un Kosovo multiétnico.

Teniendo en cuenta todo lo expuesto anteriormente, no es difícil concluir que después de 1999 Kosovo ha tenido problemas para satisfacer las diferentes expectativas, tanto a nivel local como internacional. Sin embargo, incluso si la falta de capacidad para satisfacer algunas de las pautas de referencia promovidas inicialmente llegará a ser menos problemático con el tiempo $^{51}$, se requerirá tarde o temprano un status claro, de modo que las relaciones regionales e internacionales de Kosovo, así como membresías puedan ser definidas. Como correctamente se ha observado por Ker-Lindsay y Economides, "donde la cuestión del status de Kosovo se vuelve más compleja y se convertirá en un problema importante será cuando Kosovo llegue a una etapa en la que esté dispuesto a establecer una relación más formal con la Unión Europea, como la conclusión real de una Acuerdo de Asociación y Estabilización formal. Aunque esto parece que acabará siendo un largo camino a recorrer, pues si la actual situación de estancamiento en la UE persiste entonces las opciones de Kosovo de unirse a la UE aparecerán como bloqueadas, ${ }^{, 52}$.

\section{Conclusión}

En contraste con las expectativas dominantes, la solución de la cuestión de Kosovo ha sido bastante lenta, dejando a las partes interesadas desconcertadas. Con una debatida proclamación de independencia y sin un status final largamente esperado, es difícil hablar sobre el futuro de Kosovo. Como demuestra el artículo, además de las divisiones que caracterizan las posiciones serbias y las albanesas de Kosovo, los europeos tampoco han podido ofrecer una posición común y una estrategia capaz de acomodar a los serbios y a los albaneses de Kosovo, y, en general, a toda la región de los Balcanes Occidentales, si bien esta estrategia implica algunos compromisos dolorosos. Esta es un área donde la pregunta sobre la voluntad sincera de la UE de apoyar la creación de un Kosovo independiente adquiere su plena vigencia. Sin embargo, al analizar la situación en mayo de 1999, por tanto antes del final de los bombardeos de la OTAN en Serbia, un autor observó lo siguiente: "Serbia va a pagar un alto precio por su intento de subordinación y la expulsión de la mayoría abrumadora de la población de Kosovo de su provincia. En el corto plazo, es probable que se produzca un aumento en el número de refugiados desplazados de Kosovo. A medio plazo habrá enormes costos por la reconstrucción de la infraestructura de Serbia y Kosovo. Es probable que se forme una Gran Albania, como se temía por la Antigua República Yugoslava de Macedonia y Grecia. A largo plazo, el bombardeo de la OTAN sin la utilización de fuerzas de tierra pondrá de manifiesto la necesidad de un ejército europeo si hay que disuadir el nacionalismo agresivo sin recurrir a la destrucción asociada a la guerra aérea. Mientras Estados Unidos se aleja de Europa, los Estados de Europa tendrán que ir más allá de los cálculos de interés nacional para hacer valer un interés común paneuropeo en un medio donde las dos fronteras y

\footnotetext{
${ }^{51}$ Ver: Comisión Europea: "Communication from the Commission to the European Parliament and the Council on a Feasibility Study for a Stabilisation and Association Agreement between the European Union and Kosovo*”, Bruselas, 10 de octubre de 2012, COM(2012)602 final.

52 Ker-Lindsay, James \& Economides, Spyros: "Standards before Status before Accession: Kosovo's EU perspective”, Journal of Balkan and Near Eastern Studies, vol. 14, no. 1 (2012), pp. 77-92, p. 85.
} 
grupos estén protegidos y abierto a cambios acordados por los afectados, como en el caso de la reunificación alemana",53.

Teniendo en cuenta las predicciones anteriormente descritas, podemos estar de acuerdo en que Serbia ha estado pagando un alto precio por la no concesión a Kosovo de un status independiente y por lo tanto retrasar su propio progreso hacia la adhesión a la UE. Como correctamente se predijo, en el corto plazo los serbios han dejado cada vez más sus enclaves, viéndolos como un territorio extranjero en el que no se sienten seguros ya. Los que se han quedado se dieron cuenta de que deben estar dispuestos a cooperar y participar en las instituciones de Kosovo; como se explica en otro lugar, "esto se debe al hecho de que, debido a su propia situación económica lamentable, agravada por la actual crisis económica mundial, Serbia, simplemente no tiene los recursos -o no los pondrá a disposición- para apoyar a los enclaves del sur económicamente" 54 . Además, la presencia del factor albanés en Macedonia y Grecia se ve a menudo como sinónimo de inestabilidad y se discute en el contexto de la Gran Albania, una ambición lanzada en 1878 por la Liga de Prizren, un movimiento político albanés orientado a la defensa de la nación albanesa ${ }^{55}$. En cuanto a las predicciones a largo plazo, el gobierno de Bruselas tendrá que establecer una posición común y no moverse de ella, de lo contrario se requerirá la participación de otras partes. Las inconsistencias en curso dentro de la propia UE sugieren que "la participación activa de los Estados Unidos será fundamental. Washington aún es percibido por los kosovares como su respaldo internacional principal" 56 .

Hoy en día, media década después de la proclamación de la independencia, cuando se le pregunta a los líderes albaneses que evalúen la situación en Kosovo, tienden a decir que Kosovo es un Estado indivisible de grandes oportunidades ${ }^{57}$, mientras que algunos otros informes han demostrado claramente que la situación general en Kosovo es más bien inestable y que muchos jóvenes no ven ningún futuro allí y, de hecho, le gustaría marcharse ${ }^{58}$. Huelga decir que muchos observadores han realizado importantes esfuerzos para elaborar y recomendar políticas que, en su opinión, funcionarían mejor, de forma que la cuestión de Kosovo se acabara resolviendo y se abriese un nuevo capítulo en la historia de Kosovo ${ }^{59}$, pero lo que todos ellos tienden a subrayar es el hecho de que la única solución duradera será la que complazca tanto a los serbios como a los albaneses de Kosovo, en lugar de alienarles aún más. Teniendo esto presente, el Acuerdo de abril 2013 entre Belgrado y Pristina realizado con la intermediación de la UE - calificado como "un acuerdo histórico" por los representantes de la $\mathrm{UE}^{60}$, "la mejor oferta posible" por Ivica Dacic, el primer ministro de Serbia 61 , "un primer e

\footnotetext{
${ }^{53}$ Brewin, Christopher: "Should NATO Bomb Serbia?”, en Waller, Michael, Drezov, Kyril \& Gökay, Bülent (eds.) (2001): Kosovo: The Politics of Delusion, London, Frank Cass, pp. 83-89, p. 88.

54 Džihić, Vedran \& Kramer, Helmut (2009): Kosovo After Independence: Is the EU's EULEX Mission Delivering on its Promises?, Berlin, Friedrich-Ebert-Stiftung, p. 9.

${ }^{55}$ Ver, por ejemplo: Austin, Robert C.: "Greater Albania: The Albanian State and the Question of Kosovo, $1912-$ 2001”, en Lampe, John R. \& Mazower, Mark (eds.) (2004): Ideologies and National Identities: The Case of Twentieth Century Southeastern Europe, Budapest, Central European University Press, pp. 235-253; Canak, Jovan (ed.) (1998): Greater Albania: Concepts and Possible Consequences, Belgrade, Institute of Geopolitical Studies; Kola, Paulin (2003): In Search of Greater Albania, London: C. Hurst \& Co.

${ }^{56}$ Lehne, Stefan: "Kosovo and Serbia: Toward a Normal Relationship", Carnegie Policy Outlook, marzo 2012, en http://carnegieendowment.org/files/Kosovo_and_Serbia.pdf.

57 “Jahjaga: Kosovo država velikih mogućnosti”, Tanjug, 16 de febrero de 2013, en http://www.b92.net/info/vesti/index.php?yyyy=2013\&mm=02\&dd=16\&nav_category=640\&nav_id=687511.

58 "Mladi bez perspektive na Kosovu", Deutsche Welle, 16 de febrero de 2013, en http://www.b92.net/info/vesti/index.php?yyyy=2013\&mm=02\&dd=16\&nav_category=640\&nav_id=687394.

${ }^{59}$ Ver, por ejemplo: International Crisis Group: "Kosovo and Serbia: A Little Goodwill Could Go a Long Way", Europe Report, no. 215, 2 de febrero de 2012.

${ }^{60}$ Unión Europea: "Serbia and Kosovo Reach Landmark Deal", 19 de abril de 2013, en

http://eeas.europa.eu/top_stories/2013/190413_eu-facilitated_dialogue_en.htm.
} 
histórico acuerdo entre Serbia y Kosovo" por Hashim Thaçi, el primer ministro de Kosovo ${ }^{62}$, y como "una oportunidad histórica para mejorar la protección de los derechos humanos" por Human Rights Watch ${ }^{63}$ - podría ser considerado como un nuevo capítulo en la historia de Kosovo, proporcionando espacio para reconsiderar políticas y, de hecho, realizar nuevos ajustes políticos y conseguir soluciones duraderas.

61 "Dačić: Najbolja ponuda dosad, međunarodno priznat sever", Politika, 20 de abril de 2013, en http://www.politika.rs/rubrike/tema-dana/Dacic-Ocekujem-konsenzus-o-ponudi-iz-Brisela.lt.html. 62 "Majorities in Kosovo, Serbia Support New Deal", Deutsche Welle, 20 de abril de 2013, en http://www.dw.de/majorities-in-kosovo-serbia-support-new-deal/a-16759798.

${ }^{63}$ Human Rights Watch: "Serbia-Kosovo: Landmark Opportunity for Human Rights", 23 de abril de 2013, en http://www.hrw.org/news/2013/04/23/serbiakosovo-landmark-opportunity-human-rights. 